\title{
Statistical Analysis of Conductor Motion in LHC Superconducting Dipole Magnets
}

\author{
M. Calvi, N. Ponomarev, P. Pugnat, and A. Siemko
}

\begin{abstract}
Premature training quenches are usually caused by the transient energy release within the magnet coil as it is energized. The dominant disturbances originate in cable motion and produce observable rapid variation in voltage signals called spikes. The experimental set up and the raw data treatment to detect these phenomena are briefly recalled. The statistical properties of different features of spikes are presented like for instance the maximal amplitude, the energy, the duration and the time correlation between events. The parameterization of the mechanical activity of magnets is addressed. The mechanical activity of full-scale prototype and first preseries LHC dipole magnets is analyzed and correlations with magnet manufacturing procedures and quench performance are established. The predictability of the quench occurrence is discussed and examples presented.
\end{abstract}

Index Terms-Conductor motions, LHC, quench training, spikes, statistics, superconducting magnets.

\section{INTRODUCTION}

$\mathbf{T}$ HE Large Hadron Collider (LHC) now under construction at CERN in Geneva Switzerland will provide protonproton collisions with a center-of-mass energy of $14 \mathrm{TeV}$. In order to achieve this design energy within the constraint of the $27 \mathrm{~km}$ LEP tunnel, the main bending magnets must provide a dipole field of $8.33 \mathrm{~T}$. The choice of NbTi superconducting cables operating at $1.9 \mathrm{~K}$ was made and the main dipoles were designed to have the superconducting limit at $9.76 \mathrm{~T}$.

Like most large scale magnets, the LHC dipoles exhibit training quenches i.e., a progressive improvement of the field level reached after repeated quenching. This type of premature quenches mainly originate from local energy deposition due to conductor motion or epoxy resin cracking. All of these mechanical events produce in general a rapid variation of the voltages, called spikes, across the magnet coil and in the so-called quench antennas [1]. The location, the time distribution and the energy of such precursors to quench contain useful information concerning the mechanical stability of the

Manuscript received October 21, 2003.

M. Calvi is with the European Laboratory for Nuclear Research, CERN, Geneva, CH-1211 Switzerland. He is also with the Departement de Physique de la Matiere Condensee, Geneva University, 24 quai Ernest-Anserment, Geneva, CH-1211, Switzerland (e-mail: marco.calvi@cern.ch).

N. Ponomarev is with the European Laboratory for Nuclear Research, CERN Geneva, CH-1211 Switzerland. He is also with the Joint Institute for Nuclear Research and Scientific Centre of Applied Research, Joliot-Curie 6, 141980 Dubna, Russia (e-mail: nikolay.ponomarev@ cern.ch).

P. Pugnat and A. Siemko are with the European Laboratory for Nuclear Research, CERN, Geneva, CH-1211 Switzerland (e-mail: pierre.pugnat @ cern.ch; andrzej.siemko@cern.ch).

Digital Object Identifier 10.1109/TASC.2004.829053 superconducting coils. In order to perform a statistical study, the development of dedicated software tools for data treatment were undertaken [2].

The production of the preseries LHC superconducting main dipoles started in 2000. The main cold test results, including quench performance, are presented in [3]. In this article, the dedicated experimental tools used during cold tests of superconducting magnets will be briefly recalled. Then the software development required to identify and discriminate mechanical events will be summarized. Examples of results coming from power tests of the LHC preseries dipoles and analyzed with these software tools will be presented. The statistical distributions of relevant parameters of recorded spikes will be analyzed in view of a better understanding of the mechanical stability of the coil structure. Finally, the exploitation of such development for quench performance analysis of LHC superconducting magnets will be discussed.

\section{EXPERIMENTAL}

\section{A. Voltage-Taps and Quench-Antenna Techniques}

The localization of premature quenches using pick-up coils [4] also called Quench-Antennas (QA), and Voltage-Taps (VT) are in daily use for quench analysis [3], [5]. Using the same techniques the signals occurring well before quenches can also be studied [2].

The shafts designed for the magnetic measurements are used as QA. They are composed of 13 identical segments and cover the two apertures of 15-meter long LHC dipoles with an array of pick-up coils [1]. Segments are made of a ceramic tube containing three identical tangential search coils each having a length of $1.116 \mathrm{~m}$. The acquisition system records signals coming both from VT and QA. It is triggered by the quench detectors used for magnet protection. Several compensation schemes, which connect pick-up coils in pairs are used to cancel the noise coming mainly from the power converter and the natural vibration of ceramic tubes. The later was found to produce negligible effects for frequencies higher than $2 \mathrm{kHz}$, which are of importance for this study. The maximum sampling frequency of the multi-channel transient recorder is $50 \mathrm{kHz}$ per channel. The transient recorder can be used in multi-event mode to record spikes that occur during the current ramping up to a quench. To record a maximum number of pre-quench spikes with a sufficient time resolution, $5 \mathrm{kHz}$ recording was used and the pre-trigger time was set to $9 \mathrm{~s}$. 


\section{B. Software Development}

1) Signal Treatment: Programs dedicated to the analysis of spikes were developed using LabVIEW on a Sun workstation [2]. Three main steps of the data treatment can be put forward. The first one concerns the extraction of characteristic signals after noise rejection (identification). The second one is related to the association of spikes coming from the same event (globalization). The third steps is the parameterization of individual spikes to calculate all statistical parameters needed to characterize general features and evolution of the mechanical activity of magnets during tests.

Apart from methods of association, both channel groups for QA and VT were treated identically. So far, more attention was paid to QA channels, which record more events than VT and provide information about spike distribution along the axis of the magnet. As two compensation schemes are used, every QA channel provides a differential measurement either inside the magnet section or between different sections. To avoid ambiguities and problems of redundancy, only the first kind of QA channels is treated for the moment. The channels recorded at $5 \mathrm{kHz}$ during $9 \mathrm{~s}$ before the quench, were scanned independently to detect spike signals as narrow peaks or oscillations exceeding the level of background noise characterized within each channel by its standard deviation $\sigma$. The $5 \sigma$ criterion was used in this study but this number can be used as a free parameter. Every spike was then recorded as a set of parameters reflecting its channel identity, location, time, amplitude and shape.

A single mechanical motion can produce spike signals in several channels. To count the real number of independent events, the globalization procedure is applied. It groups coincident spikes (i.e., spikes whose starting time difference is less or equal to the sampling time interval) detected inside the same magnet element (aperture or section) into a "local spike". Each of them can be associated to a common mechanical event. The second part of the globalization procedure applied to QA channels is to consider the possible sequential propagation of the mechanical motion from initial to neighboring sections (the typical propagation velocity measured for spikes is between 1 to $2 \mathrm{~km} / \mathrm{s}$ ).

As a result every registered "local spike" obtains a "global spike" number for identification. It is then possible to analyze the signature of every mechanical motion and to characterize its features. All results are finally stored as files and are ready for statistical analysis which can be performed with different software tools.

2) Graphical User Interface: A graphical user interface using ROOT [6], [15] objects was developed to visualize the various statistical parameters relevant to the analysis of the mechanical activities of magnets prior to their quenches. The program is structured as two dynamic lists (Fig. 1). The first list is in the form of a tree (magnet builder, magnet name and measurement name) and is linked to the database in which all information about measurements are available. In the second list the analysis of the selected measurements are stored and it is linked to a canvas where all histograms are available for visual inspection and selection of a particular analysis. This

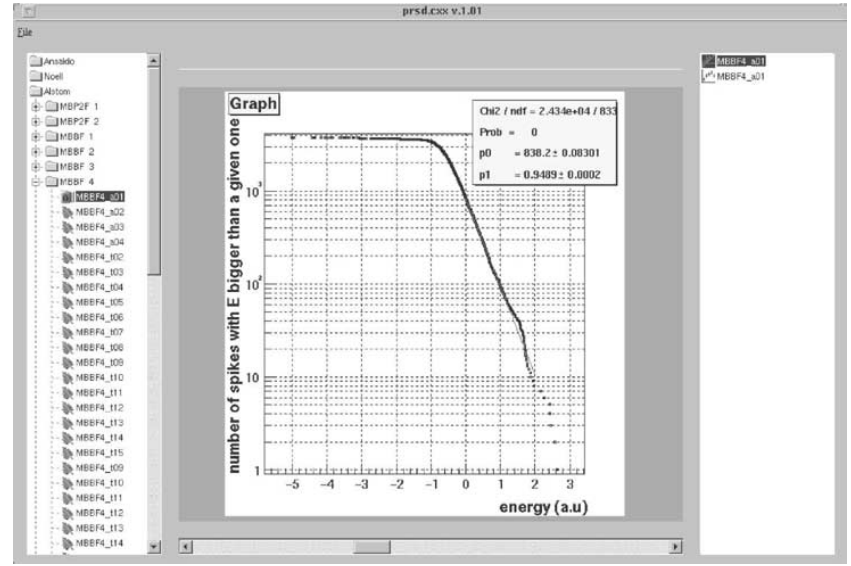

Fig. 1. The graphical user interface dedicated to the statistical treatment of the extracted from raw measurements spikes.

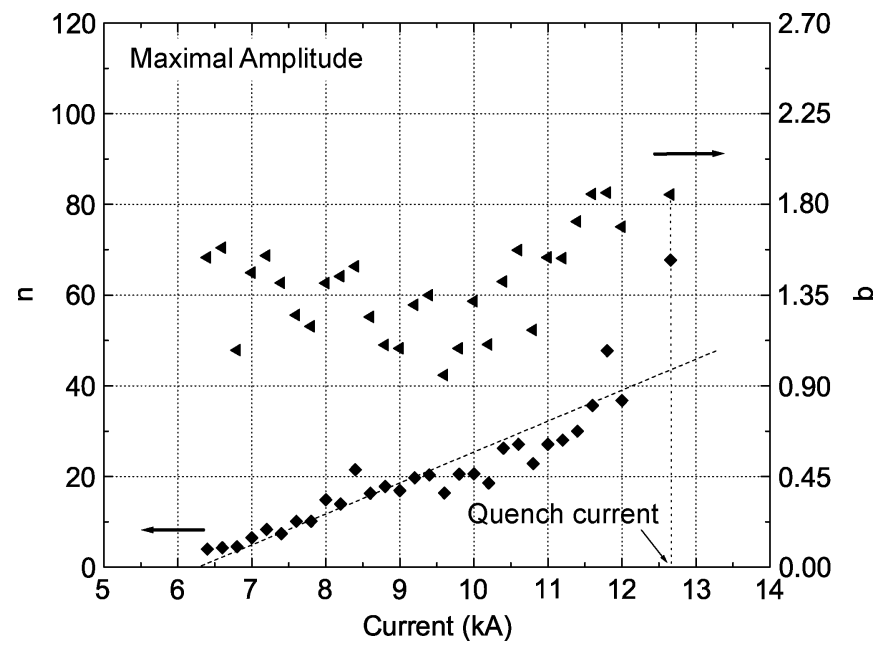

Fig. 2. Parametric study of the maximal amplitude during the whole ramp to predict its quench performance.

general approach gives the opportunity to compare different analyzes for different magnets and to upgrade easily the data treatment at any time.

\section{DATA ANALYSIS}

Within the standard measurement program for the LHC dipoles each magnet is energized at the nominal current ramp rate and all signals are recorded $9 \mathrm{~s}$ before the quench. To analyze these data it is important to know the magnet powering history. When a magnet is powered for the first time its mechanical activity is visible from low current level (about $4 \mathrm{kA}$ ) as it is shown in the example of Fig. 2. If a magnet is energized reaching a level $I_{\max }$ without quenching and afterward the current is ramped down, the mechanical activity is no longer visible in the following current ramp till the level $I_{\max }$ is reached. This simple experiment shows that the magnets have a memory of the previous current cycles. If a quench previously occurred the memory effect is still present but the behavior is not so simple and clear as for the previous case. The memory 


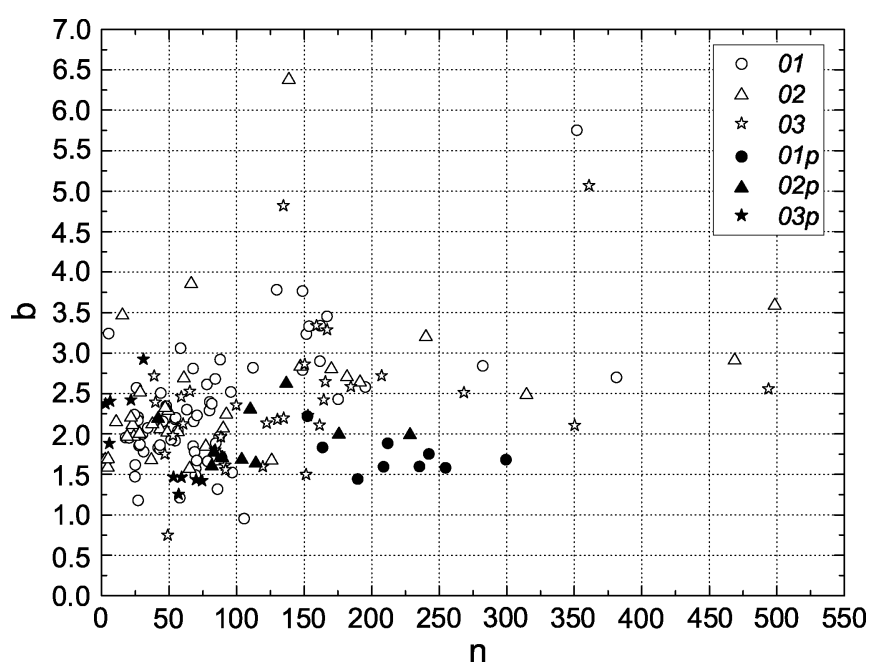

Fig. 3. The results of the power law parametric analysis of the spikes, obtained for the reference amplitude $A_{0}=1 \mathrm{mV}$ and observed in preseries $(01,02,03)$ and prototype $(01 p, 02 p, 03 p)$ LHC dipole magnets.

and consequently the non reproducibility of the experiments makes the statistical treatment of the data more critical because it is not possible to repeat the experiments. As a consequence the statistical objects that are described in the following are to be considered as a static picture of an irreversible and dynamic phenomenon, like for instance in the statistics of earthquakes [2], [7].

The main parameters chosen to describe the mechanical activity in a magnet are the maximum amplitude and the energy of a spike. The cumulative distributions of these parameters are evaluated in order to avoid the problem of choosing a bin size. This distribution has not been normalized to one but to the number of spikes per unit time:

$$
P(x)=\frac{\Delta N}{\Delta t} \int_{x}^{+\infty} p(\xi) d \xi,
$$

where $\Delta N$ is the total number of global spikes detected during the observation time $\Delta t(9 \mathrm{~s})$ and $\mathrm{x}$ is the normalized parameter (for example $x=A / A_{0}$, where $A$ and $A_{0}$ are the spike amplitude and the reference spike amplitude, respectively). Equation (1) gives the number of spikes per unit time which has a parameter bigger than a given value. This approach has been used to save the information related to the time dependent phenomena.

\section{A. Parameterization of the Mechanical Activity}

To reduce the information a power law is used to fit [2] the cumulative distribution (1) (between 1 and 10):

$$
f(x)=n \cdot x^{-b},
$$

where $n$ is the number of spikes per unit time with $x$ bigger than one and $b$ is a positive number which characterize the probability decay of $\mathrm{x}$ : the bigger is $\mathrm{b}$ the lower is the probability to observe an event characterized by $x$. If $\mathrm{b}$ is less than one the mean value is not defined and if $b$ is less than two the sigma is not defined. The power law distribution is also endowed with
TABLE I

EXAMPLE of ANALYSIS FOR PRESERIES AND PROTOTYPES LHC SUPERCONDUCTING MAGNETS

\begin{tabular}{lccccc}
\hline \hline Builder & $\langle n\rangle$ & $\sigma_{n}$ & $\langle b\rangle$ & $\sigma_{b}$ & Number of quenches \\
\hline 01 & 84.6 & 71.12 & 2.3 & 0.75 & 67 \\
02 & 100.5 & 119.8 & 2.43 & 0.9 & 35 \\
03 & 143.5 & 100.5 & 2.38 & 0.84 & 33 \\
$01 \mathrm{p}$ & 255.7 & 128.27 & 1.77 & 0.25 & 10 \\
$02 \mathrm{p}$ & 116.5 & 53 & 1.95 & 0.34 & 10 \\
$03 \mathrm{p}$ & 38.2 & 27.9 & 1.9 & 0.58 & 10 \\
\hline \hline
\end{tabular}

a remarkable property, known as "self-similarity" or "scale invariance": the ratio of the probability of two values is only a function of the ratio of these variations. This simple approach describes the mechanical activity of a magnet with a point in a bidimensional parameter space. On the contrary it is not always the best fit for certain measurements. Several parametric distributions have been taken into consideration as possible candidates but even if they were suitable in several cases the power law was found to be the most general with lowest number of parameters.

\section{B. Example of Training Quench Analysis}

The dynamics of the training is followed in the $\mathrm{n}$ versus $\mathrm{b}$ space. In Fig. 3 the results of the parameterization for the preseries of dipole magnets is presented. The mean and the standard deviation for $\mathrm{n}$ and $\mathrm{b}$ have been evaluated and are shown in Table I. Via those parameters it is possible to define an ensemble of points which is grouped around the mean values with a spread of $\sigma$ which represent the general trend. All the magnets which have been found out of this region have shown a low quench performance. Conversely some troublesome magnets have been found inside.

For the preseries dipole magnets exhibiting a good quench performance it is no longer possible to clearly identify a particular magnet builder, as was the case for prototypes. This suggests that the procedures applied to wind the coils and assemble the cold mass are getting homogeneous among the firms, at least with respect to this particular analysis.

\section{Predicting the Quench Performance}

To improve the statistical predictability of the quench performance special tests have been performed. The global mechanical activity has been investigated for several current levels before the quench and both $\mathrm{n}$ and $\mathrm{b}$ have been plotted as a function of the current (Fig. 2). The parameter $\mathrm{n}$ increases linearly with the current until the quench level is approached. The value of $\mathrm{n}$ which corresponds to the $9 \mathrm{~s}$ before the quench is clearly out of the linear trend. A new mechanical regime seems to be correlated with the origin of the quench and this was observed for several magnets. Recording spikes during powering brings useful information about the quench performance and can be used as the standard test to check mechanical stability of the magnet production.

\section{Fundamental ASPECTS}

An explanation of the possible mechanism of the training behavior of superconducting magnets is presented in [8], where 
the dynamics of the compaction of the magnet winding is discussed using cellular automaton. The main idea is the competition between the compaction induced by the Lorentz forces and the decompaction of the structure induced by the local thermal expansion, which follows a quench.

The stick-slip mechanism and the power-law distribution of spike amplitudes have suggested to investigate several existing models developed originally to provide a theoretical base to the statistics observed for earthquakes world wide. The sand-pile model [9] has been studied with the aim to explain in a qualitative way the quench training and related phenomena. This model is not the best candidate for a more qualitative analysis of the quench training in superconducting magnets. The Burridge-Knopoff model of earthquake in its differential equation formalism [10] and in its cellular automaton formalism [11], [12] is closer to the magnet system because the dynamics are deterministic and determined by an external force with the total number of particles conserved during the whole process. The two-dimensional BK model [13] seems to be the best candidate to describe the mechanical activity of magnets because the exponent of the power law is not an universal constant and is a function of the conservation level of the energy exchange between particles. Also a two-dimensional nonlinear BK model [14] is available in which it is possible to parameterize the elasticity of the materials involved.

All models described above have the disadvantage of having a dynamics which strongly depends on boundary conditions. The interesting power law distribution which is the signature of long-range correlation among the particles of the system is destroyed if the system has no open boundary conditions. In the sand-pile model an unstable boundary particle is ejected outside the system and lost. This feature is a fundamental ingredient to observe the long-range correlations. Similarly in the BK model the last element in the direction of the force is supposed to move and the system is undergoing a constant translation with a mean velocity. In such a model the boundary conditions are not relevant because the characteristic dimensions of blocks are not an active ingredient of the dynamics of the system. In the nonlinear model the boundary conditions start playing a role and the long-range correlations are preserved only if the boundary blocks are free to move.

\section{Future DEVElopMEnT}

A new acquisition system with larger memory is planned to be used. This system will allow to investigate the memory effect after a quench, i.e., the competition between stabilization induced by the Lorentz forces and the instabilities induced via thermal expansion.

A new quench antenna has been designed and the first prototype will be soon available. This device has been dedicated to investigate the mechanical activity in the magnet heads, which are known weak points where almost all quenches occur. This device will allow precise localization of the starting region of a quench with possibility to distinguish between different mechanical disturbances.

\section{CONCLUSION}

The new measurement system introduced in [2] has been intensively used during the test of the preseries LHC superconducting magnets. With this technique a good reproducibility of the coil winding and magnet assembly procedures have been observed among the three companies producing the LHC dipoles. This was far to be the case during the previous prototyping phase. Several magnets which were refused due to their insufficient quench performance have been found out of the reproducibility area in the $b$ versus $n$ plane. The statistical quench performance predictability has not yet been observed for all low-performance magnets because the statistics is describing collective effects and the behavior of a superconducting magnet may be driven by a single but extreme event. Preliminary results suggest that the statistical predictability of the quench performance may be improved by increasing the recording time and the correlation between mechanical regimes and the quench field level.

\section{ACKNOWLEDGMENT}

The authors would like to thanks Prof. Flukiger for his constant support to this project, G. D'Angelo and his colleagues for the devotion in testing of superconducting magnets and R. Brun and his team for constant support with ROOT.

\section{REFERENCES}

[1] J. Billan et al., "Twin rotating coils for cold magnetic measurements of $15 \mathrm{~m}$ long LHC dipoles," IEEE Trans. Appl. Superconduct., vol. 10, no. 1, pp. 1422-1426, March 2000.

[2] P. Pugnat, B. Khomenko, A. Rijllart, S. Sanfilippo, and A. Siemko, "Statistical diagnosis method of conductor motions in superconducting magnets to predict their quench performance," IEEE Trans. Appl. Superconduct., vol. 11, no. 1, pp. 1705-1708, 2001.

[3] A. Siemko, L. Bottura, P. Pugnat, L. Rossi, S. Sanfilippo, and J. Vlogaert, presented at the Quench Performance and Field Quality of the LHC Preseries Cryodipoles, this conference.

[4] D. Leroy, J. Krzywinski, V. Remondino, L. Walckiers, and R. Wolf, "Quench observation in LHC superconducting magnets by field perturbation measurements," IEEE Trans. Appl. Superconduct., vol. 3, pp. 781-784, 1993.

[5] L. Bottura, P. Pugnat, A. Siemko, J. Vlogaert, and C. Wyss, "Performance of the LHC final design full scale superconducting dipole prototypes," IEEE Trans. Appl. Superconduct., vol. 11, no. 1, pp. 1554-1557, 2001.

[6] R. Brun and F. Rademakers, "ROOT—an object oriented data analysis framework," in Proceeding AIHENP96 Workshop Lausanne, 1996.

[7] D. Sornette, Critical Phenomena in Natural Science. Heidelberg: SPRINGER, Springer Series in Synergetics, 2000.

[8] P. Pugnat, M. Calvi, and A. Siemko, presented at the Training Behavior of Accelerator Superconducting Magnets, this conference.

[9] P. Back, C. Tang, and K. Wiesenfield, "Self-organized criticality," Phys. Rev. A, vol. 38, no. 1, pp. 364-374, 1988.

[10] J. M. Carlson and J. S. Langer, "Mechanical model of an earthquake fault," Phys. Rev. A, vol. 40, no. 11, pp. 6470-6484, 1989.

[11] H. Nakanishi, "Cellular-automaton model of earthquakes with deterministic dynamics," Phys. Rev. A, vol. 41, no. 12, 1990.

[12] — "Statistical properties of cellular-automaton model for earthquakes," Phys. Rev. A, vol. 43, no. 12, 1991.

[13] Z. Olami, H. J. Feder, and K. Christensen, "Self-organized criticality in continuous, nonconservative cellular automaton modeling earthquakes," Physical Review Letters, vol. 68, no. 8, 1992.

[14] K. Leung, J. Muller, and J. V. Andersen, "The Two-Dimensional Nonlinear Burridge-Knopoff Model of Earthquakes,", arXiv:cond-mat/9510020 v2, 1995.

[15] R. Brun and F. Rademakers, "ROOT—an object oriented data analysis framework," Nucl. Inst. and Meth. in Phys. Rev. A, vol. 389, pp. 81-86, 1997. 